\section{Rehabilitación estética y funcional en paciente con compromiso gastroesofágico y desorden temporomandibular: reporte de un caso}

\author{
Aesthetic and functional rehabilitation in a \\ patient with gastroesophageal compromise \\ and temporomandibular disorder: a case \\ report
}

\begin{abstract}
Resumen
Paciente masculino, de 32 años, acudió a la Clínica de Prótesis Parcial Fija (PPF), de la Facultad de Odontología de Araçatuba-Universidad Estadual Paulista, manifestando como queja principal que sus dientes anteriores estaban "feos y rotos". Después del examen clínico, el paciente fue diagnosticado con desorden temporomandibular, bruxismo y presencia de erosiones dentarias (intrínsecas y extrínsecas). Se propuso el acompañamiento de un médico gastroenterólogo y la rehabilitación con prótesis libres de metal $(11,12,21$ y 22) confeccionadas en silicato de litio reforzado con zirconio por el sistema CAD/CAM. Después de la endodoncia de los incisivos antero-superiores, fueron instalados los pernos de fibra de vidrio anatomizados con resina compuesta; la impresión fue realizada con hilo retractor y silicona de adición. Después de la prueba estética y ajustes oclusales, las PPFs de e-max Ceram, fueron preparadas para cementación resinosa con el sistema Variolink II color Light. Después de 1 semana, se tomó la impresión de la arcada superior y posteriormente se le instaló una placa miorrelajante. El paciente y los profesionales involucrados aprobaron el resultado final, comprobando la eficacia estética asociada al uso de prótesis libre de metal, a pesar de que el paciente presenta compromiso gastroesofágico y desorden temporomandibular.
\end{abstract}

Palabras clave: Rehabilitación bucal; Cerámica; Estética dental; Cementación (fuente: DeCS BIREME).

\begin{abstract}
A 32-year-old male patient attended in the Fixed Partial Prosthesis Clinic (PPF), of the Araçatuba Dental School-Paulista State University, stating as the main complaint that his anterior teeth were "ugly and broken". After clinical examination, the patient was diagnosed with temporomandibular disorder, bruxism and the presence of dental erosions (intrinsic and extrinsic). It was proposed to be followed up by a gastroenterologist and rehabilitation with metal-free prostheses $(11,12,21$ and 22) made of lithium silicate reinforced with zirconium by the CAD / CAM system were proposed. After endodontics of the anterior-superior incisors, the anatomized fiberglass posts with composite resin were installed; the impression was made with retractor wire and addition silicone. After aesthetic testing and occlusal adjustments, the e-max Ceram PPF"s were prepared for resin cementation with the Variolink II color Light system. After 1 week, the impression of the upper arch was taken and subsequently a muscle relaxant plate was installed. The
\end{abstract}

\section{Caso Clínico}

Marcio Campaner 1,a, Juliana Lujan Brunetto 1,a, Caroline de Freitas Jorge e ${ }^{1, b}$, Rosse Mary Falcón-Antenucci ${ }^{2, c}$, Aldiéris Alves Pesqueira ${ }^{1,0}$

${ }^{1}$ Universidade Estadual Paulista "Júlio de Mesquita Filho", Faculdade de Odontologia de Araçatuba (FOAUNESP), Araçatuba, São Paulo, Brasil

${ }^{2}$ Universidad Inca Garcilaso de la Vega, Instituto de Investigación (UIGV), Lima, Perú.

a Magister en Odontología.

${ }^{b}$ Cirujano Dentista.

${ }^{\mathrm{C}}$ Doctor en Odontología.

\section{Correspondencia:}

Aldiéris Alves Pesqueira: aldieris.pesqueira@unesp.br Rua José Bonifácio 1193, Vila Mendonça, Araçatuba-Brasil, CEP: $16015-050$.

ORCID: 0000-0003-3020-5253

\section{Coautores:}

Marcio Campaner: marciocampaner17@gmail.com ORCID: 0000-0002-8750-0555

Juliana Lujan Brunetto: juliana_brunetto@hotmail.com ORCID: 0000-0001-9518-695X

Caroline de Freitas Jorge: carolinefreitas48@gmail.com ORCID: 0000-0001-5372-7164

Rosse Mary Falcon-Antenucci: rossefalcon@gmail.com ORCID: 0000-0002-6184-0981

Editor:

Donald Ramos-Perfecto

Universidad Nacional Mayor de San Marcos, Perú.

Conflicto de intereses: Rosse Mary Falcon-Antenucci es miembro del comité editor de la revista Odontología Sanmarquina.

Fuente de financiamiento: autofinanciado

Recibido: 26/02/21

Aceptado: $12 / 03 / 21$

Publicado: 01/04/21

( Los autores. Este artículo es publicado por la revista Odontología Sanmarquina de la Facultad de Odontología, Universidad Nacional Mayor de San Marcos. Este es un artículo de acceso abierto, distribuido bajo los términos de la licencia Creative Commons Atribución 4.0 Internacional (CC BY 4.0) [https://creativecommons.org/ licenses/by/4.0/deed.es] que permite el uso, distribución y reproducción en cualquier medio, siempre que la obra original sea debidamente citada de su fuente original. 
patient and the professionals involved approved the final result, verifying the aesthetic efficacy associated with the use of a metal-free prosthesis, despite the fact that the patient has gastroesophageal involvement and temporomandibular disorder.

Keywords: Oral rehabilitation; Ceramics; Dental Aesthetics; Cementation (source: MeSH NLM).

\section{Introducción}

La demanda por tratamientos estéticos ha aumentado progresivamente en la Odontología, sin embargo, la rehabilitación de una sonrisa antiestética en la región anterior maxilar es un desafío clínico ${ }^{1}$. Los aspectos de la belleza son muy subjetivos y están influenciados principalmente por la cultura, la moda, las emociones, la edad y la historia individual, pero las características geométricas generales de un rostro, que dan lugar a la percepción de la belleza, pueden ser universales. Por ello, en la búsqueda de encontrar una composición agradable en la sonrisa, se pueden aplicar algunos factores como: proporción, simetría y perspectiva ${ }^{2-4}$.

Además de los aspectos estéticos y funcionales, la rehabilitación estética anterior por medio de prótesis fija, involucra aspectos sociales, en los que mejora la perspectiva del paciente respecto a su autoestima y se siente más cómodo al relacionarse con los demás ${ }^{5}$. Así, el cirujano dentista también debe satisfacer las expectativas y deseos emocionales de su paciente, brindándole una mejor calidad de vida, a través de la confianza, dedicación y profesionalismo ${ }^{6}$.

El éxito de la instalación de las prótesis parciales fijas, con el fin de rehabilitar al paciente, incluye una anamnesis detallada, que recoge información esencial, como la presencia de actividad parafuncional como el bruxismo, desorden temporomandibular y enfermedad por reflujo gastroesofágico (ERGE), detectando erosiones en los dientes. Además, se debe realizar una correcta planificación, delineando un plan de tratamiento adecuado para el paciente ${ }^{7-9}$.

La estimación de la prevalencia de ERGE en Brasil es de alrededor del $12 \%{ }^{8}$. La erosión dental causada por esta enfermedad se caracteriza por la disolución de la superficie del diente ${ }^{10,11}$. Los ácidos de etiología de ERGE provienen de la bulimia y del reflujo gastroesofágico ${ }^{11,12}$.

Con la evolución de los materiales para uso en Odontología se ha producido una mejora en las propiedades físicas y mecánicas de los materiales, sin embargo, la unión con la estructura de los dientes debe preservarse cuando sea necesario. La rehabilitación de las piezas dentales dañados por erosión o bruxismo puede ser hecha con resinas compuestas, pernos y núcleos de metal fundido, restauraciones metálicas fundidas y finalmente restauraciones de cerámica, siendo esta última la mejor opción en el caso de dientes desgastados y que presentan una mayor necesidad estética ${ }^{13}$. Las restauraciones totalmente cerámicas se utilizan ampliamente en la odontología, ya que pueden imitar las características ópticas del esmalte y la dentina. Además, poseen estabilidad de color a largo plazo, resistencia al desgaste, biocompatibilidad, adaptación marginal y buena relación con los tejidos periodontales ${ }^{14}$.

En el caso reportado en este trabajo, se utilizaron cerámicas reforzadas con disilicato de litio, ya que tienen una matriz vítrea, donde los cristales se encuentran dispersos de manera entrelazada, por lo que la propagación de grietas en el interior es difícil ${ }^{15}$. Además, este tipo de cerámica es altamente estética, porque el índice de refracción de la luz es similar al del esmalte dental, sin una interferencia significativa de translucidez, lo que permite reproducir la naturalidad de la estructura dental ${ }^{16}$.

El objetivo de este trabajo fue exponer el reporte de un caso clínico de rehabilitación estética con coronas libres de metal en las piezas 11, 12, 21 y 22, confeccionadas en cerámica de silicato de litio reforzado con zirconio por el sistema CAD/CAM en un paciente con ERGE y bruxismo.

\section{Reporte del caso}

Paciente masculino de 32 años acudió a la clínica PPF de la Facultad de Odontología de Araçatuba, en la Universidad Estadual Paulista "Júlio de Mesquita Filho" el 2018, con la queja principal de desgaste en sus dientes anteriores. Después de la anamnesis y examen clínico detallado, se verificó un marcado desgaste en la región de los incisivos (Figura 1), durante la anamnesis el paciente refirió tener un trastorno gastroesofágico y que no estaba en tratamiento médico, se le propuso el acompañamiento de un gastroenterólogo, la rehabilitación con prótesis libre de metal en las piezas 11, 12, 21 y 22, posteriormente, la instalación de una placa miorrelajante.

Para la correcta planificación de las prótesis parciales fijas de los incisivos antero-superiores, se realizó la impresión de la arcada superior e inferior con silicona de condensación pesada Zetaplus y liviana Oranwash (Zhermack, Badiapolesine, Italia) en un solo paso, esperando el tiempo de trabajo recomendado por el fabricante. Una vez obtenidos los modelos de yeso, se montaron en un articulador y se envió al laboratorio para el encerado diagnóstico de las piezas que intervinieron en la planificación.

A partir del encerado diagnóstico (Figura 2), se realizó un mock-up para evaluar el procedimiento a realizar y también para aprobación de la estética final que sería posible lograr al finalizar la rehabilitación (Figuras $3 \mathrm{y}$ 4). Luego se propuso la cementación de pernos de fibra de vidrio en las piezas dentarias tratadas endodónticamente para la realización de prótesis libres de metal en las piezas 22, 21, 11, 12 . 


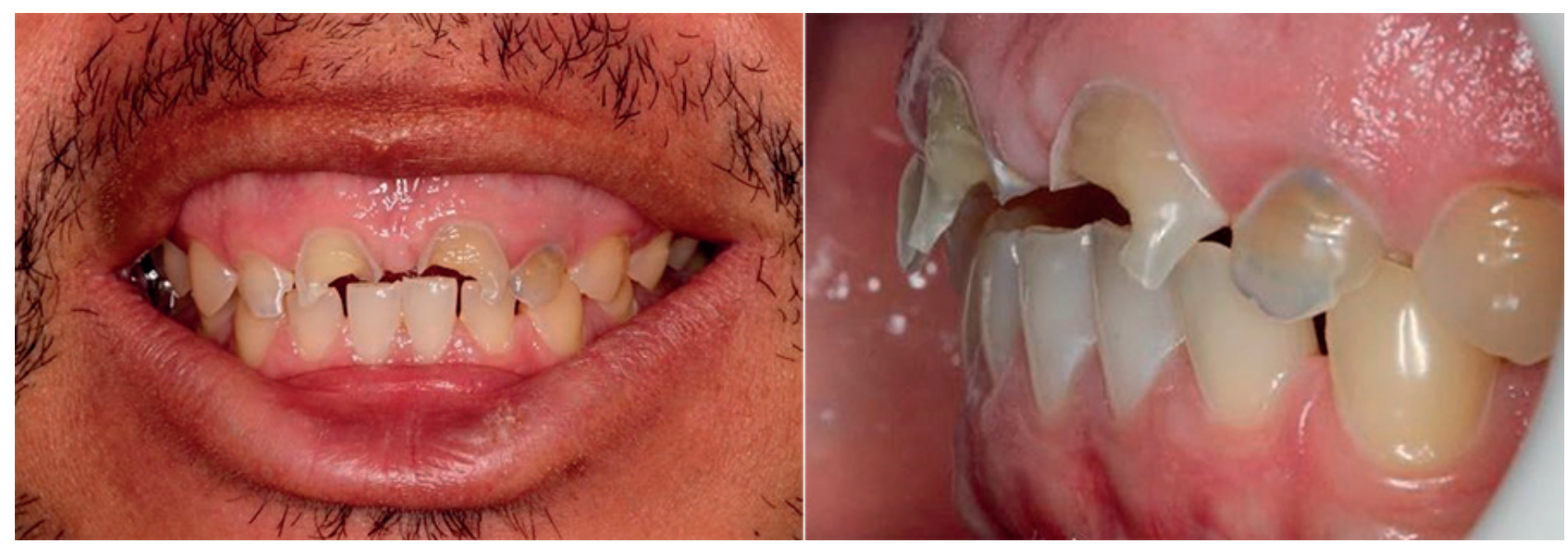

Figura 1. Sonrisa inicial

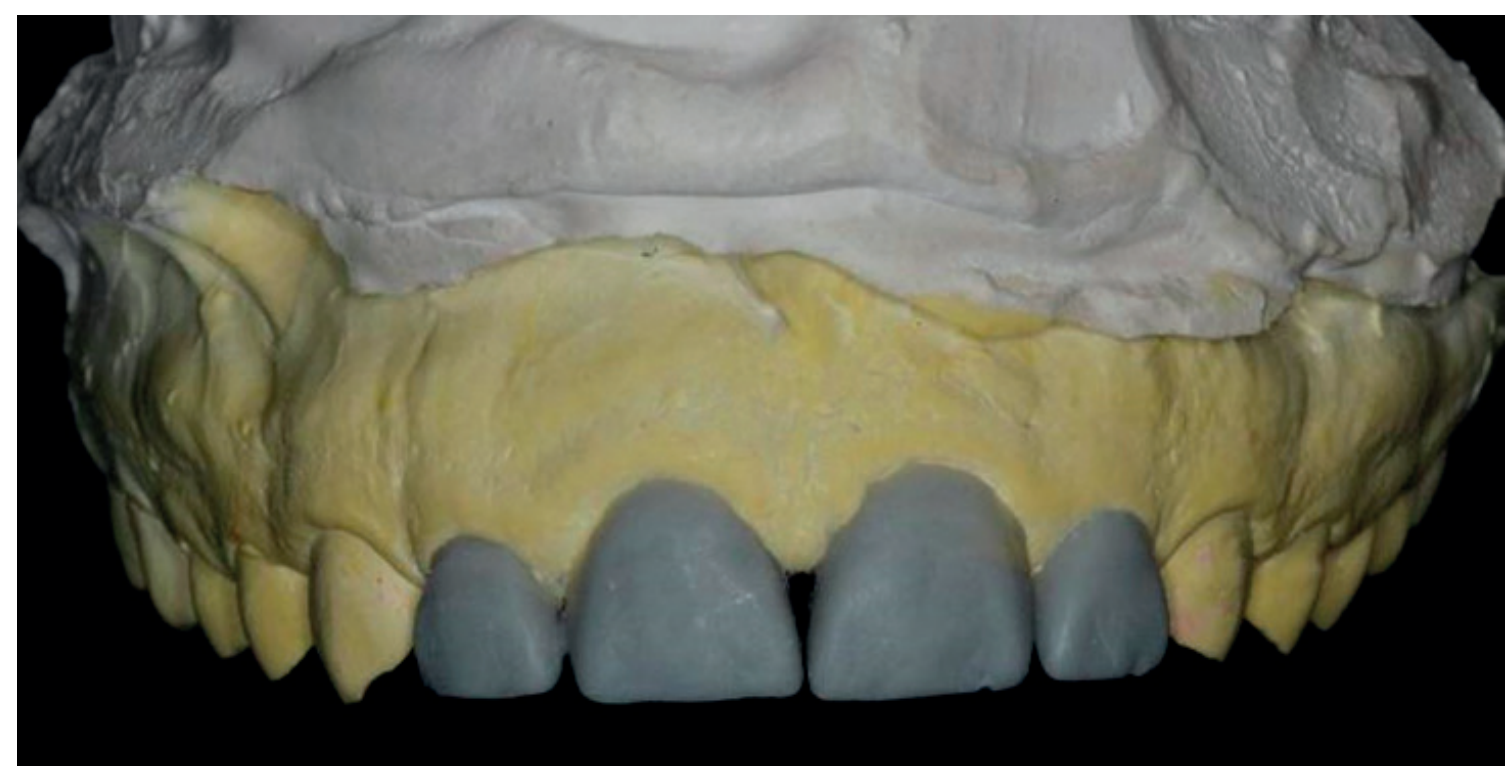

Figura 2. Encerado de diagnóstico

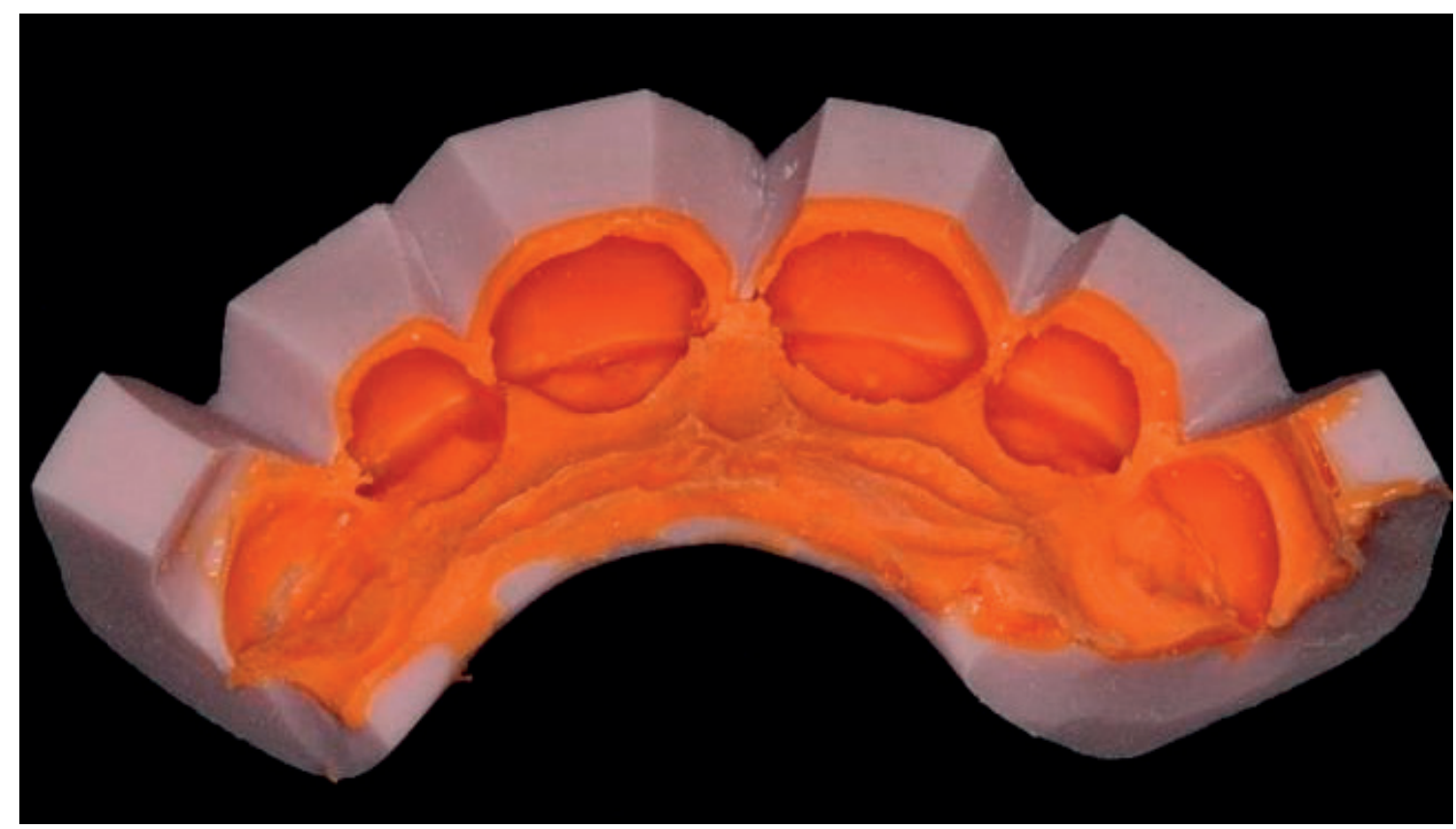

Figura 3. Guía de silicona de condensación para la realización del mock-up 


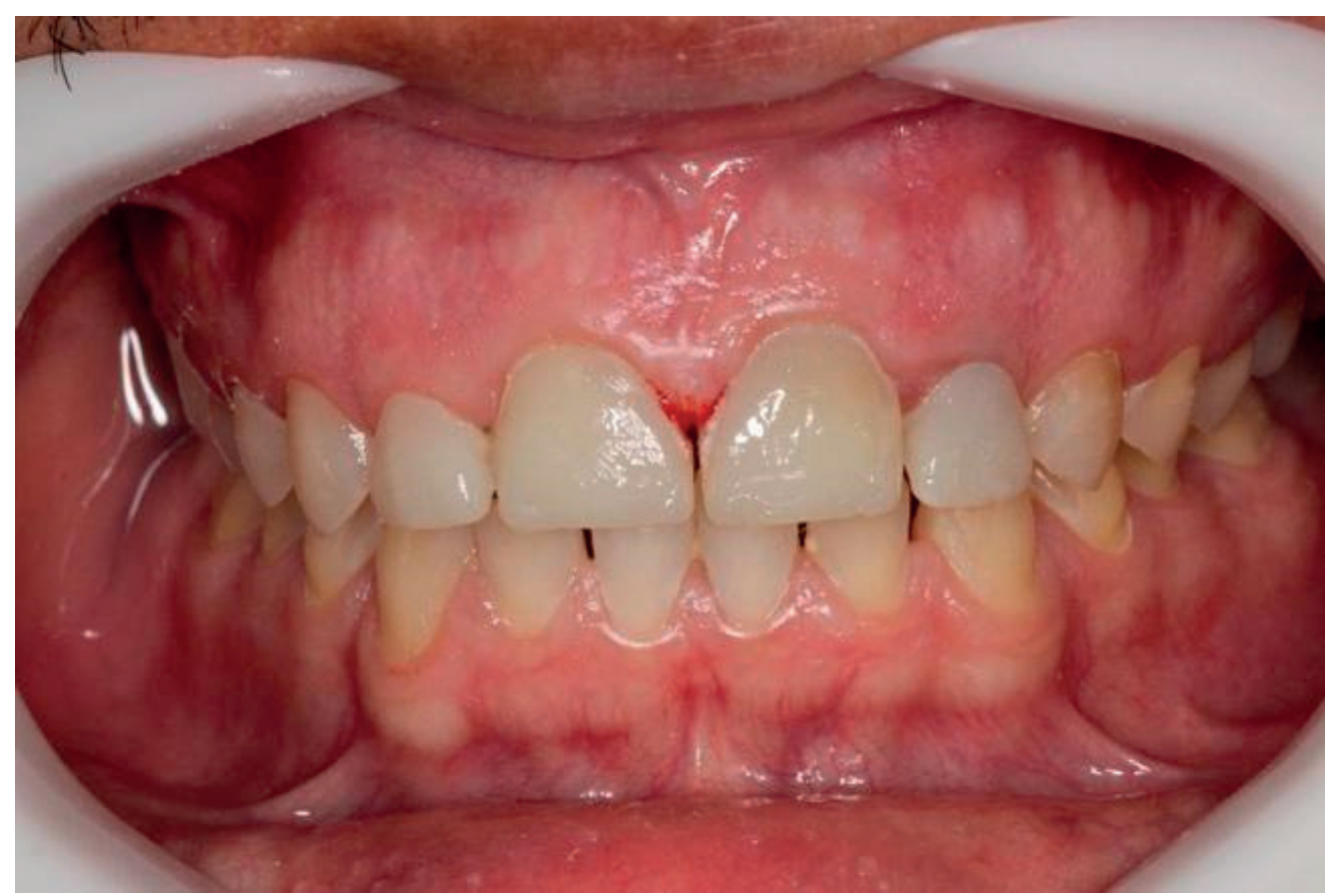

Figura 4. Prueba estética de la sonrisa (mock-up)

Se dio inicio al tratamiento realizando la desobturación de los conductos de los incisivos, todos los cuales fueron tratados endodónticamente. Por medio de radiografías periapicales fue realizada la conductometría y la desobturación con fresas Peeso (CA Maillefer - Dentsply) (Figuras 5 y 6 ).

A continuación, se cementaron los pernos de fibra de vidrio con cemento resinoso U200 (3M ESPE) (Figura 7), la reconstrucción coronaria fue realizada con resina compuesta (Z350 XT, 3M ESPE) y las preparaciones dentarias (Figura 8). Luego, se realizó la retracción gingival para tomar la impresión, se utilizó la técnica de retracción gingival mecánico-química con doble hilo retractor (Ultrapack, Ultradent, SP, Brasil), y se embebió el hilo \#000 en cloruro de aluminio (Hemostop -Dentsply Sirona, York, USA). El primero (\#000) de menor diámetro, tenía la función de reducir el flujo gingival y efectuar la retracción vertical. El segundo hilo de mayor calibre efectuó la retracción gingival horizontal. Después de la impresión, las coronas fueron confeccionadas en cerámica monolítica de disilicato de litio utilizando el sistema CAD/CAM (Figura 9), luego se realizó la prueba estética y funcional de las piezas y los ajustes oclusales (AccuFilm Carbon Paper - Parkell, NY, USA) y de punto de contacto interproximal. La elección del color se verificó a partir de la prueba húmeda, la forma y posición de los dientes, la adaptación de las prótesis y la radiografía periapical.

Con la aprobación del paciente, después de probar las piezas finalizadas, se seleccionó previamente el cemento transparente por medio de pruebas con el kit Try-In. Posteriormente, comenzó el proceso de cementación:

Preparación de los remanentes:

- Aislamiento relativo;
- Profilaxis con piedra pómez (SSWhite Duflex, RJ, Brasil) y escobilla de Robinson recta (American Burrs, SC, Brasil) en todos los pilares;

- Lavado con chorro de agua y secado con chorro de aire;

- Aplicación de ácido fosfórico (Condac 37, FGM Productos Odontológicos Ltda, SC, Brasil) en dentina por 15 segundos;

- Lavado y secado con papel absorbente;

- Aplicación del primer y adhesivo Single Bond Universal (3M, Minnesota, EUA) con la ayuda de un Microbrush $^{\circledast}$ Regular (2,0 mm);

- Fotopolimerización por 20 segundos (Bluephase NIvoclar Vivadent, SP, Brasil) en todas las caras.

Preparación de la pieza protésica:

- Condicionamiento con ácido fluorhídrico 10\% por 20 segundos;

- Lavado y secado con chorros de aire;

- Silanización con Monobond N Universal (Ivoclar Vivadent, SP, Brasil) y Microbrush ${ }^{\oplus}$ Regular (2,0 mm);

- Inserción del cemento resinoso dual Variolink ${ }^{\bullet} \mathrm{N}$ (Ivoclar Vivadent, SP, Brasil) en color Transparente con una espátula No. 1 en las paredes axiales y colocado en posición bajo presión positiva constante;

- Eliminación del exceso después de su polimerización completa, realizado con 30 segundos de fotopolimerización en cada pilar (Bluephase N - Ivoclar Vivadent, SP, Brasil). 


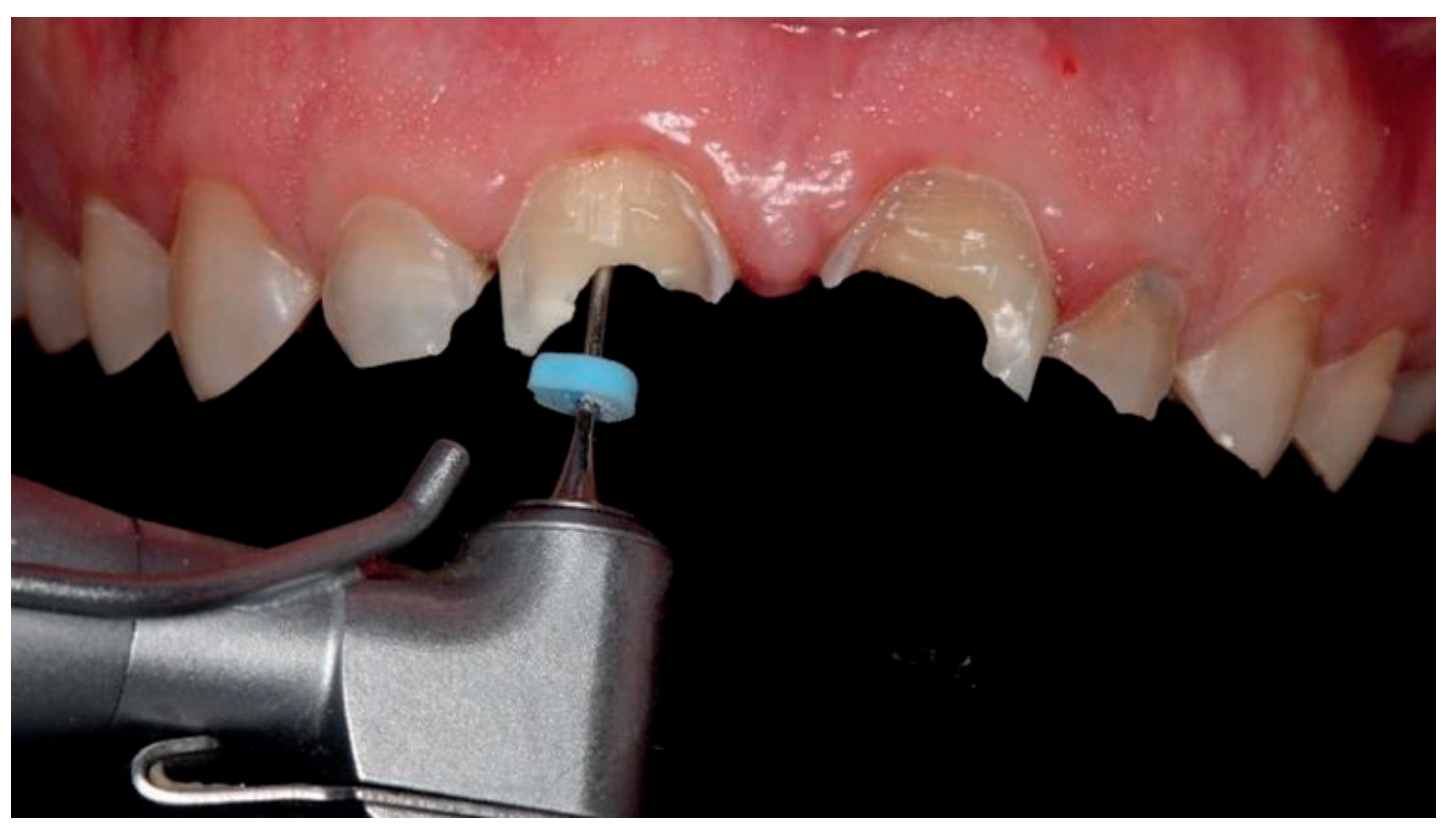

Figura 5. Desobturación de los incisivos

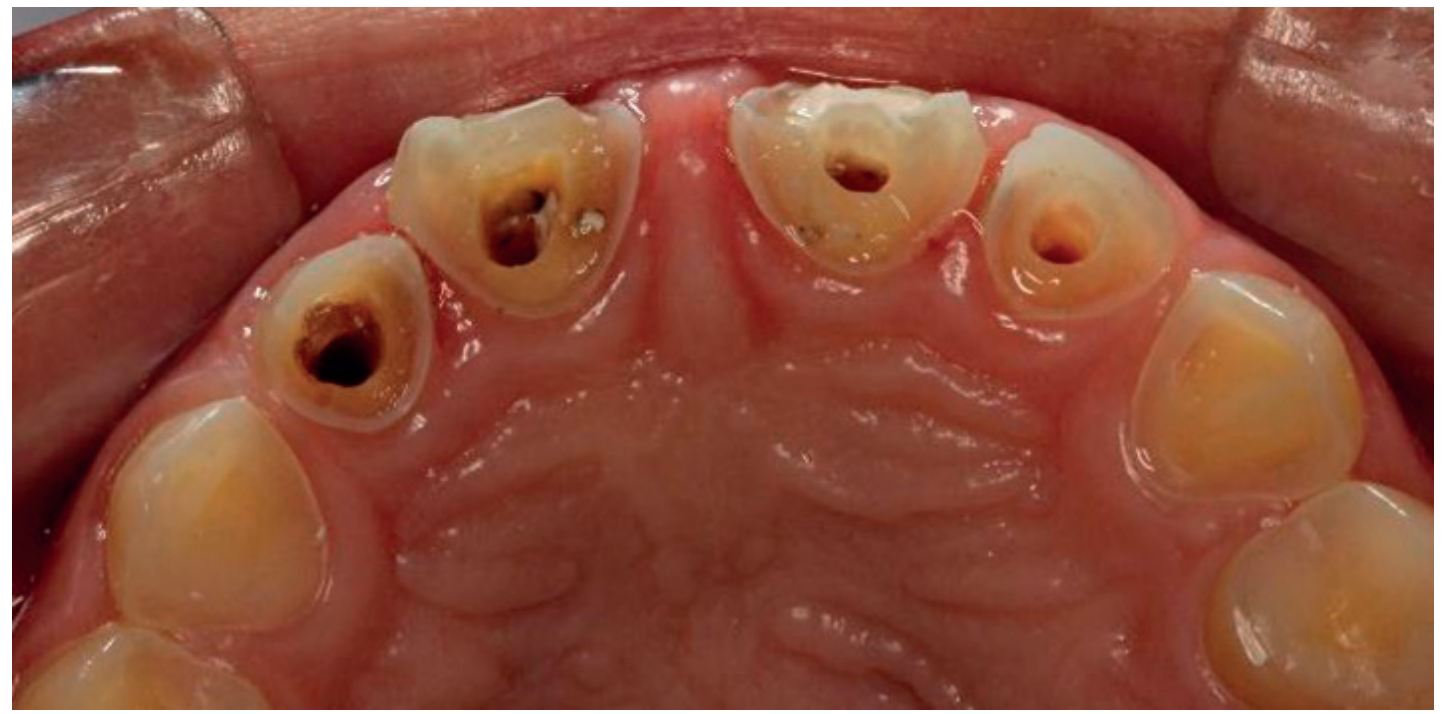

Figura 6. Vista de la arcada superior después de la desobturación

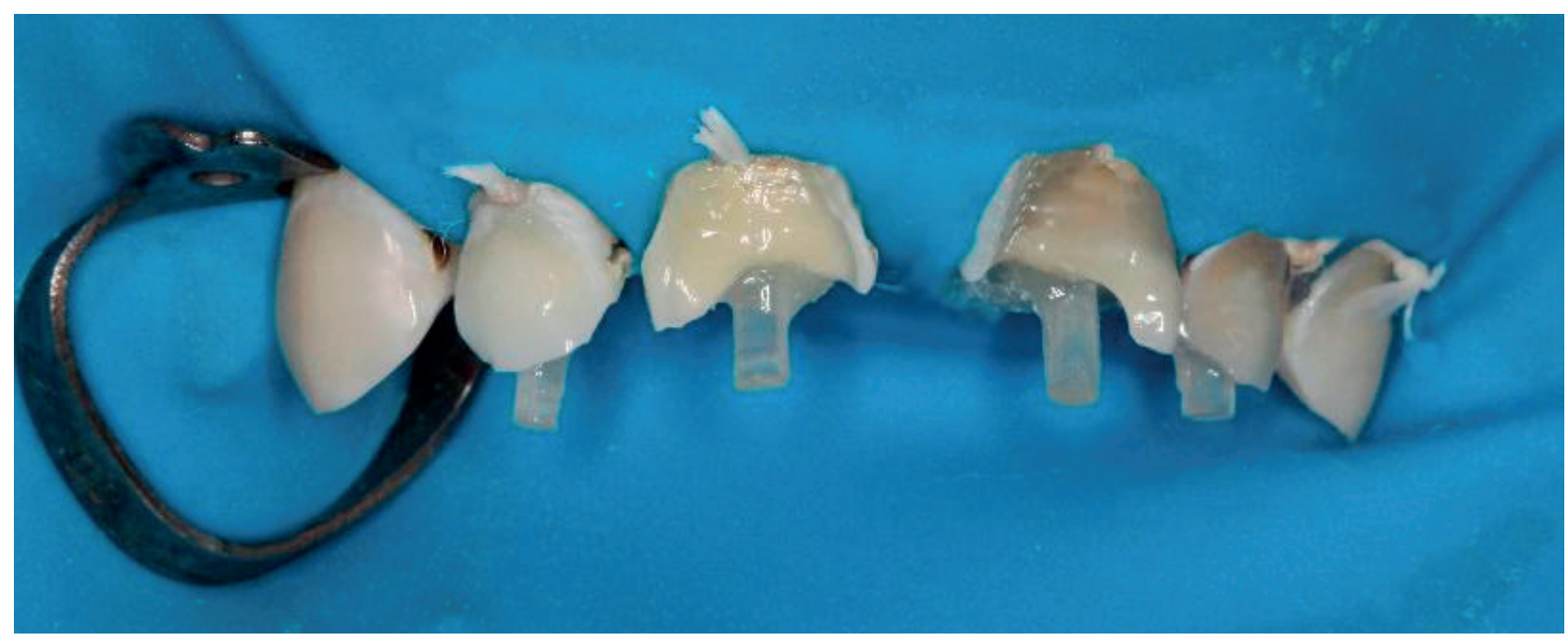

Figura 7. Cementación de los pernos de fibra de vidrio 


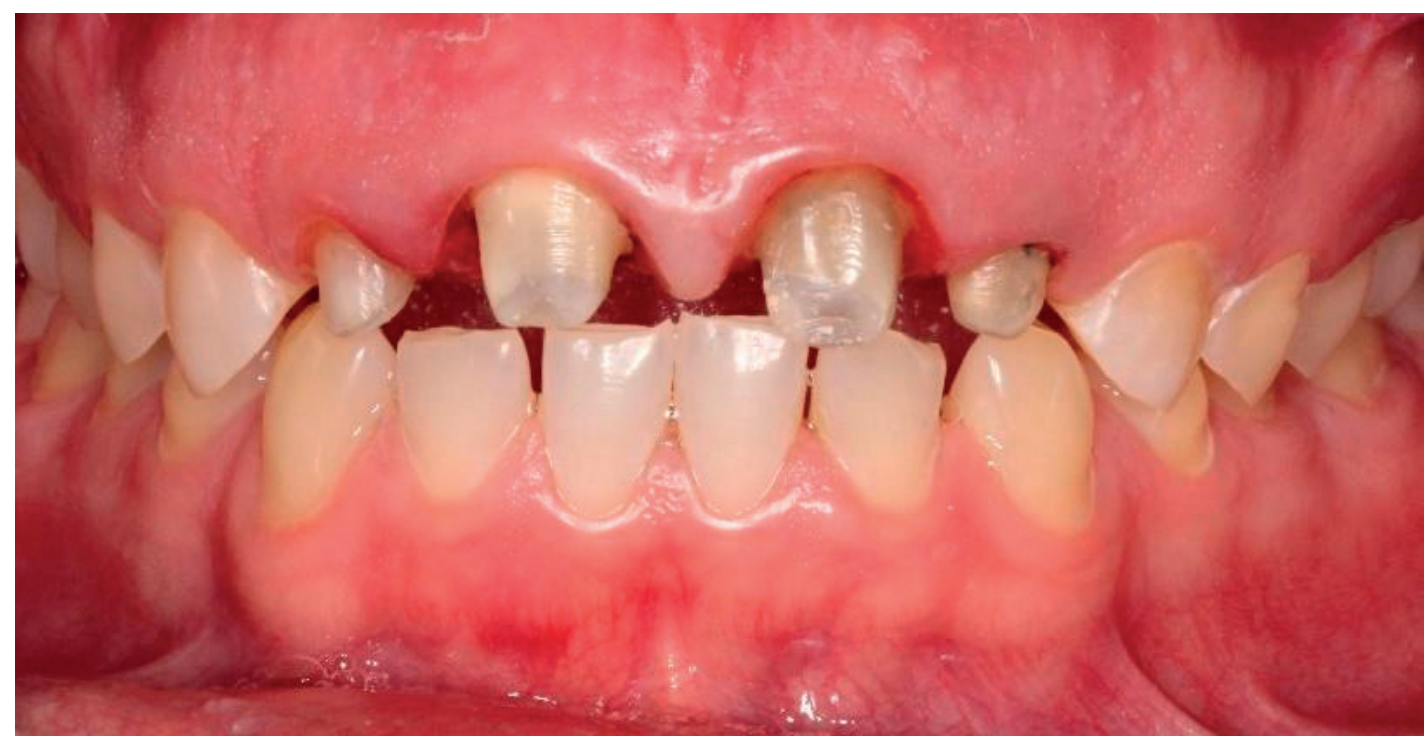

Figura 8. Vista vestibular de las preparaciones finalizadas

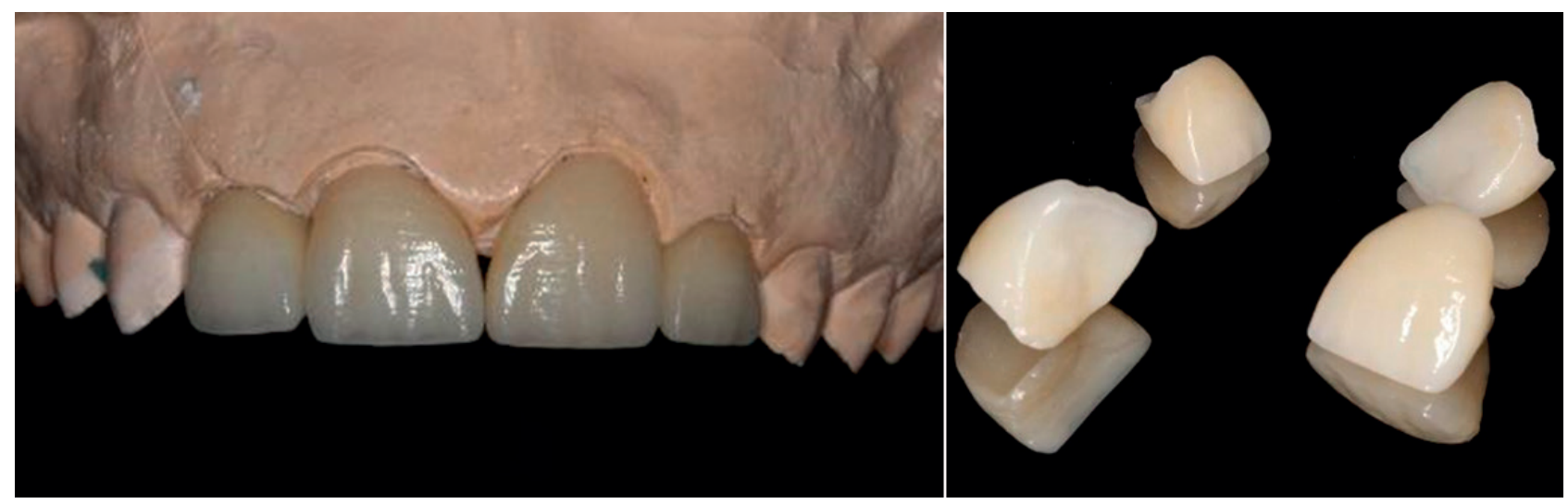

Figura 9. Aspecto final de las coronas totales de disilicato de litio

Finalizado el procedimiento de cementación (Figura 10) y a la semana de instalación (Figura 11), se realizó la impresión para la posterior instalación de la placa miorrelajante (Figura 12), para preservar el tratamiento realizado.

\section{Discusión}

Para lograr resultados funcionales satisfactorios, el examen clínico y la correcta planificación previa al tratamiento son de suma importancia. Por lo tanto, existe la necesidad de un examen clínico completo, modelos de estudio, exámenes radiográficos, además de procesos adicionales como fotografías estandarizadas, encerado de diagnóstico y mock-up, que son utilizados para ayudar a una planificación correcta, descrito en este trabajo.

El aumento en la demanda por el tratamiento rehabilitador con fin estético ha traído grandes desafíos en la odontología, porque los pacientes con complicaciones sistémicas tienen la necesidad de realizar una rehabilitación funcional y esperan una rehabilitación estética. Esto es posible gracias a la evolución de los sistemas adhesivos y materiales cerámicos que dan origen a una nueva era en la odontología ${ }^{17}$.

A partir de la correcta planificación para la realización del tratamiento, el encerado es la primera aproximación del paciente con el tratamiento a ser propuesto y a partir de la prueba del mock-up se puede realizar cambios, para que el aspecto final de las piezas presente características intrínsecas y extrínsecas de color, forma. y textura superficial de la dentición natural ${ }^{18}$. De esta manera, se tiene una mayor previsibilidad respecto a lo que será el caso finalizado. A partir del resultado del mock-up y aprobación del profesional y del paciente, tenemos una planificación más sólida, si las restauraciones finales siguen el patrón de encerado diagnóstico.

Como el tratamiento implica una rehabilitación antero-superior, el uso de pernos prefabricados reforzados con fibra de vidrio ha ganado más popularidad tanto en la clínica como en la investigación, ya que tienen un módulo de elasticidad cercano al de la estructura dentinaria, reduciendo así los riesgos de fractura radicular, y además asegura que el remanente coronario no interfiera alterando el color de las restauraciones totalmente cerámicas ${ }^{19,20}$. 


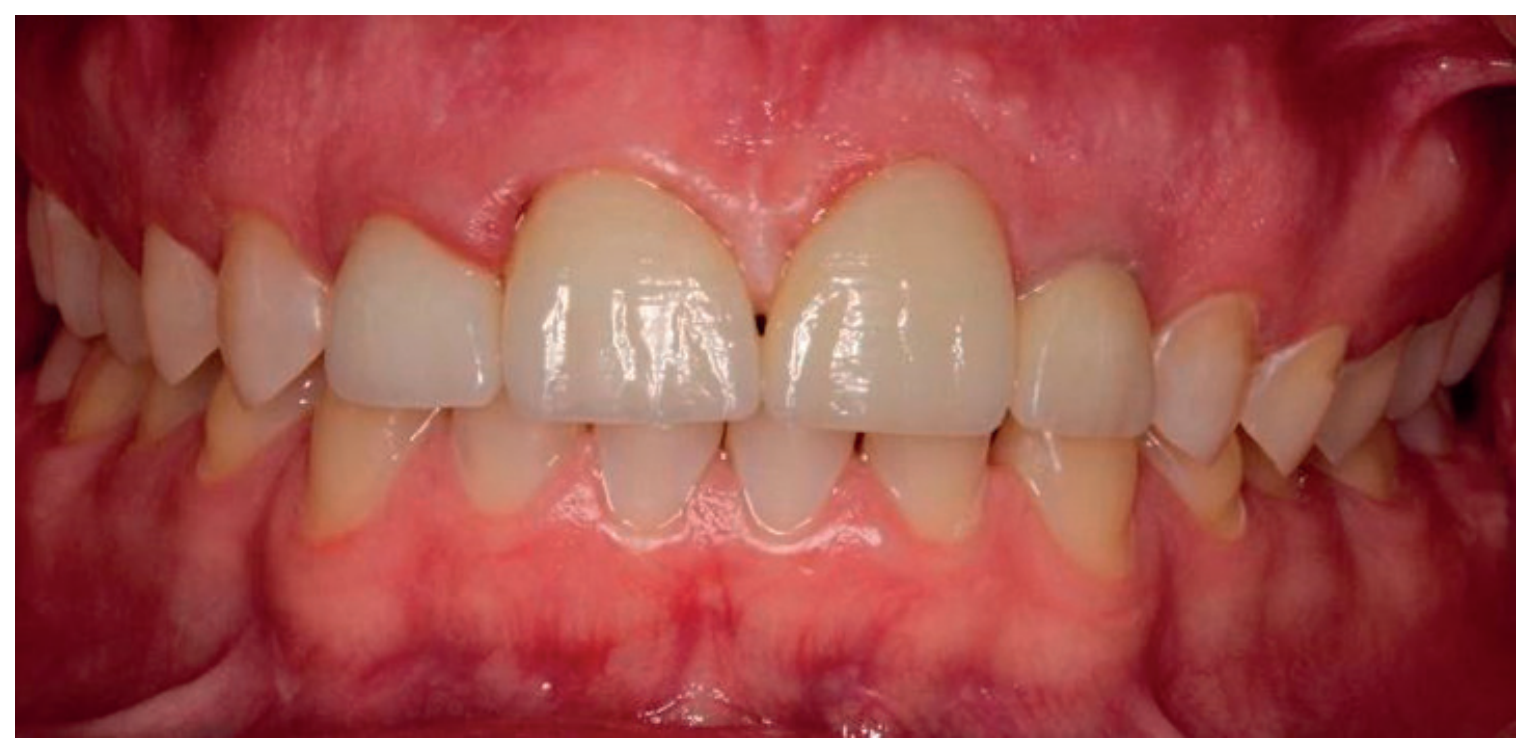

Figura 10. Aspecto final intra-oral después de la cementación

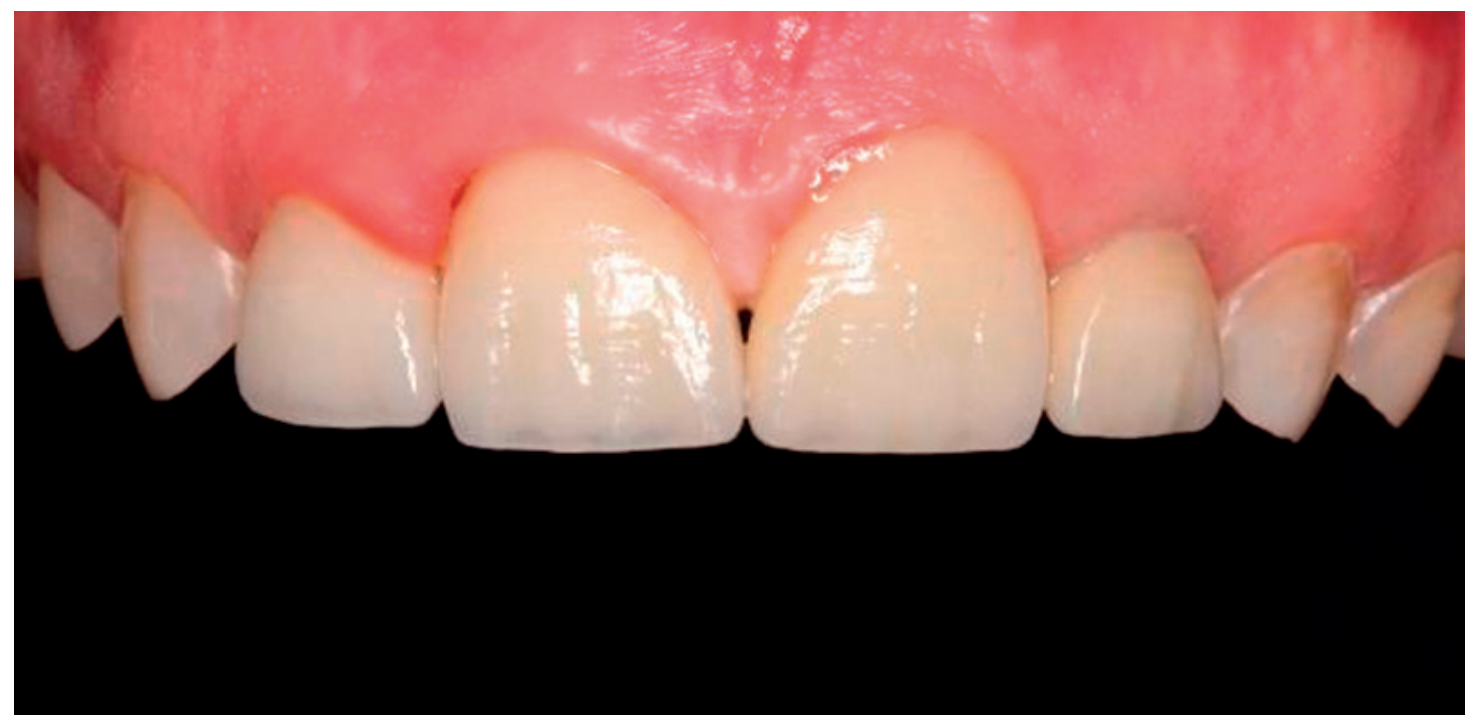

Figura 11. Aspecto final intra-oral después de 7 dias de instalación

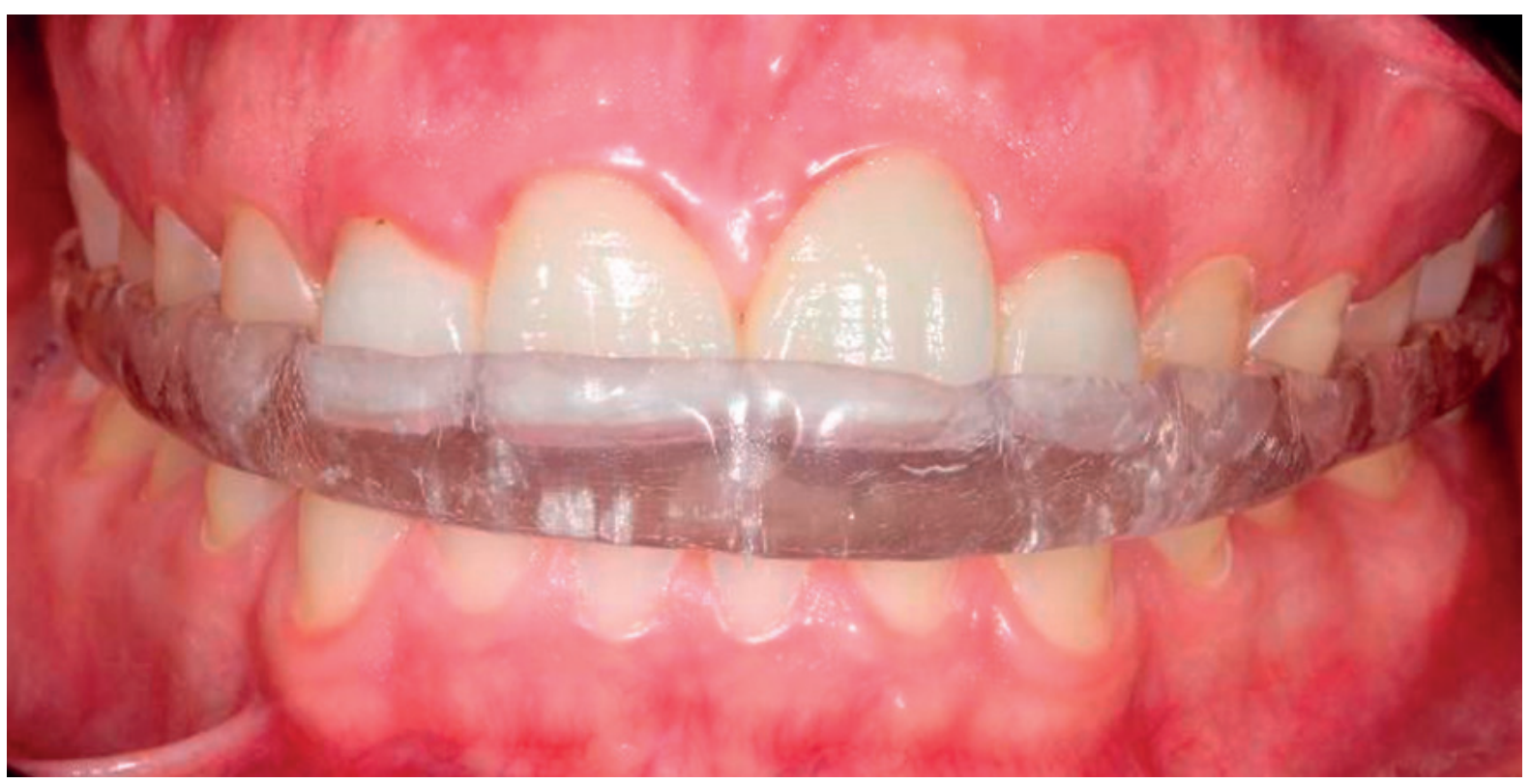

Figura 12. Instalación de la placa miorrelajante 
El IPS e.max basado en disilicato de litio tiene un mayor grado de translucidez en comparación con los sistemas basados en zirconia. Y por ello y otros estudios ${ }^{3,4,14,21}$ optamos por utilizar coronas libres de metal de disilicato de litio en el caso presentado. Este material se utiliza en la fabricación de coronas monolíticas en disilicato de litio e.max Press (Ivoclar, Vivadent) y posee varias indicaciones (por ejemplo, coronas, inlays, onlays, coronas sobre implantes y carillas) por sus propiedades estéticas y resistencia mecánica ${ }^{21}$.

Para la confección de coronas bien adaptadas, se realizó una impresión de doble hilo para una mejor retracción gingival mecánica considerando el tipo y cantidad de encía insertada (fenotipo periodontal), accediendo también al fondo del surco para el control del líquido crevicular y así lograr una impresión fiel con silicona de adición pesada y liviana.

La selección adecuada del material de cementación es un paso muy importante, y debe ser realizado desde la planificación inicial (para evitar fallas futuras) según la compatibilidad del material con la corona confeccionada. En el caso reportado, se seleccionó el sistema Variolink II. Por tratarse de un cemento resinoso, también es necesario la selección y cuidados a seguir durante la pre-cementación. La acción del cemento resinoso es una evolución en la odontología, proporcionando mejores resultados estéticos y con una excelente longevidad ${ }^{22}$.

Se realizaron controles posteriores a la instalación de las piezas, para verificar la estética, adaptación, oclusión, eficiencia de la higienización, así como la satisfacción del paciente con el tratamiento.

Con este caso se puede concluir la importancia de la correcta selección del material, teniendo en cuenta que el éxito clínico general es multifactorial y, por lo tanto, dependiente del material, del clínico y del paciente. Una planificación criteriosa permite un tratamiento estético seguro y duradero.

El paciente y los profesionales involucrados aprobaron el resultado final, demostrando la eficacia estética ligada al uso de prótesis libres de metal a pesar de la afectación gastroesofágica y el trastorno temporomandibular del paciente.

\section{Referencias bibliográficas}

1. Rodrigues RB, Veríssimo C, Pereira RD, Queiroz CL, Novais VR, Soares CJ, et al. Clareamento dentário associado à facetas indiretas em cerâmica: Abordagem minimamente invasiva. Rev Odontol Bras Central. 2012;21(11):520-5.

2. Calixto LR, Bandeca MC, Andrade MF. Enceramento diagnóstico: previsibilidade no tratamento indireto. Rev Dental Press Estét. 2011;8(3):26-37.

3. Kina S, Bruguera A. Invisível: restauraçóes estéticas cerâmicas. 2a ed. Maringá: Dental Press; 2008.

4. Amoroso AP, Ferreira MB, Torcato LB, Pellizzer EP, Mazaro JVQ, Gennari Filho H. Cerâmicas odontológicas: propriedades, indicaçôes e consideraçôes clínicas. Rev Odontol Araça. 2012;33(2):19-25.
5. Tung AW, Kiyak HA. Psychological influences on the timing of orthodontic treatment. Am J Orthod Dentofacial Orthop. 1998;113(1):29-39. DOI: 10.1016/ S0889-5406(98)70274-4.

6. Castro JDB, Silva VB. Satisfação no Atendimento Odontológico: Um Estudo na COE (Clínica Odontológica de Ensino) de Anápolis - UniEvangélica. Rev Adm. 2008;(5):34-44.

7. Pegoraro LF, Valle AL, Araujo CRP, Bonfante G, Conti PCR. Prótese Fixa. Bases para o planejamento em Reabilitação Oral. 2a ed. São Paulo: Artes Médicas; 2013.

8. Raposo NML, da Cruz MCFN, Lopes FF. A erosão dentária como uma lesấo oral predominante na doença do refluxo gastroesofágico. Rev Bras Odontol. 2010;67(2):1526. DOI: $10.18363 /$ rbo.v67n2.p.152.

9. Pace F, Pallotta S, Tonini M, Vakil N, Bianchi Porro G. Systematic review: gastro-oesophageal reflux disease and dental lesions. Aliment Pharmacol Ther. 2008;27(12):117986. DOI: $10.1111 / j .1365-2036.2008 .03694 . x$.

10. Souza CVA, Maia KD, Passos M, Wayne SC, Tunas IC. Erosáo dentária causada por ácidos intrínsecos Rev Bras Odontol. 2010;67(1):28-33. DOI: 10.18363/rbo. v67n1.p.28.

11. Mangueira DFB, Passos IA, Oliveira AFB, Sampaio FC. Erosão dentária: etiologia, diagnóstico, prevalência e medidas preventivas. Arquivos em Odontologia. 2009; 45(4):220-5.

12. Fuller JL, Johnson WW. Citric Acid Consumption and the human dentition. J Am Dent Assoc. 1977;95(1):804. DOI: 10.14219/jada.archive.1977.0551.

13. Barron RP, Carmichael RP, Marcon MA, Sandor GKB. Dental erosion in gastroesophageal reflux disease. J Can Dent Assoc. 2003;69(2):84-9.

14. Magne P, Belser U. Restauraçóes adesivas de porcelana na dentiçáo anterior: Uma abordagem biomimética. 1a ed. Rio de Janeiro: Santos Publicaçôes; 2012.

15. Kina S. Cerâmicas dentárias. Rev Dental Press Estét. 2005;2(2):111-28.

16. Guzman AF, Moore BK, Andres CJ. Wear resistance of four luting agents as a function of marginal gap distance, cement type and restorative material. Int J Prosthodont. 1997;10(5):415-25.

17. Baratieri LN, Monteiro-Júnior S. Odontologia Restauradora: Fundamentos e Possibilidades. 2a ed. Rio de Janeiro: Editora Santos; 2015.

18. Alberton SB, Alberton V, Carvalho RV. Providing a harmonious smile with laminate veneers for a $\mathrm{pa}$ tient with peg-shaped lateral incisors. J Conserv Dent. 2017;20(3):210-13. DOI: 10.4103/09720707.218311.

19. Rocca GT, Daher R, Saratti CM, Sedlacek R, Suchy T, Feilzer AJ, Krejci I. Corrigendum to Restoration of severely damaged endodontically treated premolars. The influence of the endo-core length on marginal integrity and fatigue resistance of lithium disilicate CAD-CAM ceramic endocrowns. J Dent. 2018;68:41-50. DOI: 0.1016/j.jdent.2017.10.011. 
20. Taha D, Spintzyk S, Schille C, Sabet A, Wahsh M, Salah $\mathrm{T}$, et al. Fracture resistance and failure modes of polymer infiltrated ceramic endocrown restorations with variations in margin design and occlusal thickness. J Prosthodont Res. 2018;62(3):293-7. DOI: 10.1016/j. jpor.2017.11.003.

21. Rizo-Gorrita M, Herráez-Galindo C, Torres-Lagares D, Serrera-Figallo MA, Gutiérre-Pérez JL. Biocompatibility of Polymer and Ceramic CAD/CAM Materials with Human Gingival Fibroblasts (HGFs). Polymers. 2019;11(9):1446. DOI: 10.3390/polym11091446.
22. Fonseca M. The aesthetics yearbook. 1a ed. São Paulo: Editora Napoleão; 2017. 